\title{
The Effects of Yawing Motion with Different Frequencies on the Hydrodynamic Performance of Floating Vertical-Axis Tidal Current Turbines
}

\author{
WANG Kai ${ }^{\text {a }}$, SUN Ke ${ }^{\text {a, }}$, SHENG Qi-hua ${ }^{\text {a }}$ ZHANG Liang ${ }^{\text {a }}$,WANG Shu-qi ${ }^{\mathrm{b}}$ \\ ${ }^{a}$ College of Shipbuilding Engineering, Harbin Engineering University, Harbin 150001, China \\ ${ }^{b}$ School of Naval Architecture and Ocean Engineering, Jiangsu University of Science and Technology, Zhenjiang 232000, China
}

\begin{abstract}
Under real sea conditions, the hydrodynamic performance of floating vertical-axis tidal current turbines is affected by waves and currents. The wave circular frequency is a significant factor in determining the frequencies of the wave-induced motion responses of turbines. In this study, the ANSYS-CFX software (manufacturer: ANSYS Inc., Pittsburgh, Pennsylvania, United States) is used to analyse the hydrodynamic performance of a vertical-axis turbine for different yawing frequencies and to study how the yawing frequencies affect the main hydrodynamic coefficients of the turbine, including the power coefficient, thrust coefficient, lateral force coefficient, and yawing moment coefficient. The time-varying curves obtained from the CFX software are fitted using the least-squares method; the damping and added mass coefficients are then calculated to analyse the influence of different yawing frequencies. The simulation results demonstrate that when analysing non-yawing turbines rotating under constant inflow, the main hydrodynamic coefficient time-varying curves of yawing turbines exhibit an additional fluctuation. Furthermore, the amplitude is positively correlated with the yawing frequency, and the oscillation amplitudes also increase with increasing yawing frequency; however, the average values of the hydrodynamic coefficients (except the power coefficient) are only weakly influenced by yawing motion. The power coefficient under yawing motion is lower than that under non-yawing motion, which means that yawing motion will cause the annual energy production of a turbine to decrease. The fitting results show that the damping term and the added mass term exert effects of the same level on the loads and moments of vertical-axis turbines under yawing motion. The results of this study can facilitate the study of the motion response of floating vertical-axis tidal current turbine systems in waves.
\end{abstract}

Key words: Tidal current energy; Vertical-axis tidal current turbine; Hydrodynamic performance; Added mass coefficient; Damping coefficient

\section{Introduction}

Today, traditional fossil fuel energy is the main source of energy throughout the world; however, based on current mining trends, fossil fuel reserves will become depleted within the next few years. Therefore, an increasing number of countries have begun to develop clean and renewable energy resources [1]. Tidal current energy [2] represents a type of marine renewable energy that has recently garnered interest because of its sustainability, high energy density and predictability.

Tidal current turbines are the main area of development in the field of tidal current energy generation [3-5]. Turbines can be classified as either vertical-axis turbines or horizontal-axis turbines depending on the relative orientation of the main shaft and the flow direction. Tidal current power station platforms support turbines, generators, other equipment and personnel. In deep waters, floating moored systems [6] are the best platforms for tidal current turbines because of their advantages in terms of maintenance, removal and installation. However, the floating platforms for floating tidal current power stations experience motion in six degrees of freedom induced by waves and currents. The hydrodynamic performance of turbines thus exhibits significant variations.

Research on the wave-induced hydrodynamic forces on and coefficients for floating structures continues to expand. Marine floating structures are produced in a variety of styles; however, most are too complicated for their hydrodynamic performance subject to waves and currents to be directly studied. Therefore, simple structures (such as cylinders and spheres) are used to study this problem. Yeung et al. [7] chose to study the hydrodynamic problem of a floating circular cylinder in finite-depth water by matching the eigenfunctions of the interior and exterior problems. Their paper addressed three types of radiation problems: heave, sway, and roll; then, the added mass and damping coefficients were introduced. The results were compared to study the hydrodynamic performance and revealed that the heave-added mass is logarithmic singular and that the damping coefficient approaches a constant value in the low-frequency limit. Lopes et al. [8] studied the hydrodynamic coefficients of a submerged pulsating sphere in finite-depth water based on a study by Linton. The results showed that close to the resonance frequency, both the added mass and damping coefficients for the pulsating oscillation mode are much larger than those for the heave or sway modes and that the water depth affects the damping coefficient only in the low-frequency range. The hydrodynamic performances of cylinders, spheres, and other simple structures have been studied by several 
researchers, including [9-11]. These researchers have applied the multipole method, eigenfunctions and other approaches to solve the hydrodynamic problems presented by simple geometries in waves and currents.

However, real marine structures have complex geometric shapes and present substantial difficulties in attempts to solve their corresponding hydrodynamic problems using the abovementioned theories. Computational fluid dynamics (CFD) simulations and experiments have been adopted to study the hydrodynamic performance of complex marine structures, such as floating wind turbines, tidal current turbines and floating offshore platforms, in waves and currents. Carlos et al. [12] used CFD methods and conducted experiments to calculate the hydrodynamic coefficients of heave plates for semi-submersible floating offshore wind turbines. The modelled plate diameter was $1 \mathrm{~m}$, which is the largest diameter for which data have been published. The added mass and damping coefficients, which are necessary for accurate time-domain simulations of a mooring design, were measured. Numerical simulations were also conducted following common industry standards; these simulation results were then compared with the experimental results. The research sought to improve the hydrodynamic design concept and benefit the offshore wind industry. Three-blade horizontal-axis tidal turbines were studied by Galloway et al. [13] in regular waves in deep water (wave height $=0.08 \mathrm{~m}$, inflow velocity $=1.5 \mathrm{~m} / \mathrm{s}$ ). The results showed that the average parameters (thrust and torque) were identical with and without waves but that the transient values differed greatly; the fluctuations in thrust increased by $37 \%$, and the torque increased by 35\%. Milne [14] analysed a series of experimental tests of the out-of-plane bending moment response at the root of a hydrodynamic blade to planar oscillatory motion, which was selected as an idealised representation of the unsteadiness induced by waves and turbulence. They found that for attached flow, the magnitude of the unsteady hydrodynamic contribution was relatively small compared to the loads measured under steady flow. At low tip speed ratios, the loads during dynamic stall were approximately $25 \%$ greater than those under steady flow. In 2014, Zhang et al. [15] employed the CFX software to analyse the hydrodynamic performance of a turbine ( $D=0.7 \mathrm{~m}$, $Z=2$ ) subjected to constant inflow when the turbine was experiencing forced vibration; the team studied how the hydrodynamic performance of the turbine was influenced by the surge frequency, surge amplitude and speed ratio. Then, they used the least-squares method to fit the time-varying axial force curves of a surging turbine. The damping and added mass coefficients were obtained using this method. Upon analysing these coefficients, Zhang et al. found that the surge phenomenon only weakly influenced the annual electricity output and that when the values of the surge frequency, surge amplitude and tip speed ratio increased, the oscillation amplitudes of the load coefficients also increased. However, surge motion had clear negative impacts on the structural strength and fatigue life of the turbine. Minimal research has been conducted on vertical-axis tidal turbines in which the hydrodynamic performance subject to waves and currents has been analysed. In 2011, the aerodynamic performance and wake dynamics of three different vertical-axis wind turbines were simulated in both normal and oblique positions by Scheurich et al.[16]; the results confirmed that a straight-bladed vertical-axis wind turbine that is operating in oblique flow can produce a higher power coefficient compared with when it is operating in normal flow. In 2015, A. Orlandi et al.[17] investigated a small-scale straight-bladed turbine in a wind tunnel with the rotational axis inclined between $0^{\circ}$ and $15^{\circ}$ from the vertical. They found that the power increased in skewed flows because the downwind phase of the revolution was less disturbed by the wake generated during the upwind phase. The study evaluated the influence of real motion on rotor performance. In 2015, Bedon et al.[18] used an unsteady Reynolds-averaged Navier-Stokes (URANS) CFD model to forecast the power conversion characteristics of vertical-axis wind turbine prototypes operating under tilted conditions (at tilt angles of $10^{\circ}$ and $20^{\circ}$ ), the results showed a significant decrease in power production with increasing tilt angles. The blades used by Bedon et al. were curved, unlike the straight blades used in [16,17]. Furthermore, the range of inclination angles considered by Bedon et al. was different from that of [16,17]. These differences may have been the cause of the different performances observed for turbines operating under tilted conditions.

In summary, many studies regarding simple structures have been conducted over the past several decades. Researchers are now exploring the hydrodynamic performance of marine structures subjected to waves and currents, especially 
vertical-axis current turbines, for which the rotational motion around the main axis and the wave-induced motion of the floating platforms in six degrees of freedom cause significant difficulties in such studies. Accounting for the variety of possible wave frequencies, this paper studies the effects of yawing motion with different frequencies on the hydrodynamic performance of floating vertical-axis tidal current turbines. A series of simulated operating conditions under a sequence of frequencies is analysed. The time-varying hydrodynamic curves of load and torque are measured and then fitted to obtain the damping and added mass coefficients using the least-squares fitting method. This research provides a valuable reference for the analysis of the wave-induced responses of floating support structures for tidal current turbines and for the design of electricity output control mechanisms.

\section{Numerical simulations}

\subsection{Basic modelling}

The incoming flow velocity towards the turbine is represented by $V$, and the positive direction of the $\mathrm{X}$ axis is the inflow direction. The power coefficient $C_{P}$ is the most important parameter for turbine energy conversion, and the thrust coefficient $C_{F X}$, the lateral force coefficient $C_{F Y}$, the tangential force coefficient $C_{T}$ and the normal force coefficient $C_{N}$ are essential indexes for assessing the hydrodynamic performance of a turbine. The yawing moment coefficient $C_{M}$ is specific to a turbine under yawing motion $\left(\xi=A \sin \left(\omega_{Y} t\right)\right)$.

Table 1 Nomenclature for turbines

\begin{tabular}{|c|c|}
\hline & Nomenclature \\
\hline$V$ & Incoming flow velocity \\
\hline$D$ & Turbine diameter \\
\hline$Z$ & Number of blades \\
\hline$\omega$ & Turbine angular velocity \\
\hline$\theta$ & Blade azimuth angle \\
\hline$\omega^{\prime}$ & Yawing angular velocity \\
\hline$A$ & Yawing amplitude \\
\hline $\mathrm{C}$ & Chord length \\
\hline$F_{X}$ & Turbine thrust \\
\hline$F_{Y}$ & Lateral force of turbine \\
\hline$f_{T}$ & Tangential force of blade \\
\hline$f_{N}$ & Normal force of blade \\
\hline$\lambda$ & Tip speed ratio \\
\hline$Q$ & Turbine torque \\
\hline$H$ & Blade length \\
\hline$\rho$ & Density of inflow water \\
\hline$M$ & Turbine yawing moment \\
\hline$\xi$ & Yawing angle \\
\hline$R^{\prime}$ & Length of yawing arm \\
\hline$\omega_{Y}$ & Yawing frequency \\
\hline
\end{tabular}


The following dimensionless parameters are defined:

Thrust coefficient:

$$
C_{F X}=\frac{F_{X}}{0.5 p V^{2} D H}
$$

Lateral force coefficient:

$C_{F Y}=\frac{F_{Y}}{0.5 \rho V^{2} D H}$

Power coefficient:

$$
C_{P}=\frac{Q \omega}{0.5 \rho V^{3} D H}
$$

Tangential force coefficient:

$$
C_{T}=\frac{f_{T}}{0.5 \rho V^{2} O H}
$$

Normal force coefficient:

$$
C_{N}=\frac{f_{N}}{0.5 \rho V^{2} O H}
$$

Tip speed ratio:

$$
\lambda=\frac{Q \omega}{V}
$$

Dimensionless angular velocity of yawing motion: $\quad \bar{\omega}^{\prime}=\frac{\omega^{\prime} R^{\prime}}{V}$

Dimensionless angular acceleration of yawing motion: $\quad \bar{a}^{\prime}=\frac{\dot{\omega}^{\prime} R^{\prime 2}}{V^{2}}$

Yawing moment coefficient:

$$
C_{M}=\frac{M}{0.5 \rho V^{2} D H R^{\prime}}
$$

The thrust, lateral force and yawing moment coefficients can each be divided into three components: a homogeneous hydrodynamic coefficient, a damping coefficient and an added mass coefficient.

$$
\begin{aligned}
& C_{F X}=C_{x}^{0}+n_{x x} \bar{\omega}^{\prime}+m_{x x} \bar{a}^{\prime}, C_{F Y}=C_{y}^{0}+n_{y y} \bar{\omega}^{\prime}+m_{y y} \bar{a}^{\prime}, C_{M}=C_{m}^{0}+n_{m m} \bar{\omega}^{\prime}+m_{m m} \bar{a}^{\prime} \\
& \bar{\omega}^{\prime}=\frac{\omega^{\prime} R^{\prime}}{V}=\frac{\dot{\xi} R^{\prime}}{V}=\frac{A \omega_{Y} \cos \left(\omega_{Y} t\right) \mathrm{R}^{\prime}}{V}, \bar{a}^{\prime}=\frac{\dot{\omega}^{\prime} R^{\prime 2}}{V^{2}}=\frac{\ddot{\xi} R^{\prime 2}}{V^{2}}=\frac{-A \omega_{Y}{ }^{2} \sin \left(\omega_{Y} t\right) \mathrm{R}^{\prime 2}}{V^{2}}
\end{aligned}
$$

The damping coefficients and added mass coefficients are functions of $\theta$ (the blade azimuth angle) and can be expressed as triangular functions as follows:

$$
\begin{aligned}
& n_{x x}=\frac{\delta C_{F X}}{\delta \bar{\omega}^{\prime}}=n_{x x}^{0}+\sum_{k=1}^{\infty} n_{x x}^{k} \sin \left(k z \theta(t)+\psi_{\omega^{\prime} x}^{k}\right) \\
& n_{y y}=\frac{\delta C_{F Y}}{\delta \bar{\omega}^{\prime}}=n_{y y}^{0}+\sum_{k=1}^{\infty} n_{y y}^{k} \sin \left(k z \theta(t)+\psi_{\omega^{\prime} y}^{k}\right) \\
& n_{m m}=\frac{\delta C_{M}}{\delta \bar{\omega}^{\prime}}=n_{m m}^{0}+\sum_{k=1}^{\infty} n_{m m}^{k} \sin \left(k z \theta(t)+\psi_{\omega^{\prime} m}^{k}\right)
\end{aligned}
$$

for the damping coefficients and

$$
\begin{aligned}
& m_{x x}=\frac{\delta C_{F X}}{\delta \bar{a}^{\prime}}=m_{x x}^{0}+\sum_{k=1}^{\infty} m_{x x}^{k} \sin \left(k z \theta(t)+\psi_{a^{\prime} x}^{k}\right) \\
& m_{y y}=\frac{\delta C_{F Y}}{\delta \bar{a}^{\prime}}=m_{y y}^{0}+\sum_{k=1}^{\infty} m_{y y}^{k} \sin \left(k z \theta(t)+\psi_{a^{\prime} y}^{k}\right) \\
& m_{m m}=\frac{\delta C_{M}}{\delta \bar{a}^{\prime}}=m_{m m}^{0}+\sum_{k=1}^{\infty} m_{m m}^{k} \sin \left(k z \theta(t)+\psi_{a^{\prime} m}^{k}\right)
\end{aligned}
$$

for the added mass coefficients.

Finally, the thrust, lateral force and yawing moment coefficients are expressed as follows: 


$$
\begin{aligned}
C_{F X}= & C_{x}^{0}+n_{x x} \bar{\omega}^{\prime}+m_{x x} \bar{a}^{\prime}=C_{x x}^{0}+\sum_{k=1}^{\infty} C_{x x}^{k} \sin \left(k z \theta(t)+\psi_{x x}^{k}\right) \\
& +\left\{n_{x x}^{0}+\sum_{k=1}^{\infty}\left[n_{x x}^{k} \sin \left(k z \theta(t)+\psi_{\omega^{\prime} x}^{k}\right)\right]\right\} \bar{\omega}^{\prime} \\
& +\left\{m_{x x}^{0}+\sum_{k=1}^{\infty}\left[m_{x x}^{k} \sin \left(k z \theta(t)+\psi_{a^{\prime} x}^{k}\right)\right]\right\} \bar{a}^{\prime} \\
C_{F Y}= & C_{y}^{0}+n_{y y} \bar{\omega}^{\prime}+m_{y y} \bar{a}^{\prime}=C_{y y}^{0}+\sum_{k=1}^{\infty} C_{y y}^{k} \sin \left(k z \theta(t)+\psi_{y y}^{k}\right) \\
& +\left\{n_{y y}^{0}+\sum_{k=1}^{\infty}\left[n_{y y}^{k} \sin \left(k z \theta(t)+\psi_{\omega^{\prime} y}^{k}\right)\right]\right\} \bar{\omega}^{\prime} \\
& +\left\{m_{y y}^{0}+\sum_{k=1}^{\infty}\left[m_{y y}^{k} \sin \left(k z \theta(t)+\psi_{a^{\prime} y}^{k}\right)\right]\right\} \bar{a}^{\prime} \\
C_{M}= & C_{m}^{0}+n_{m m} \bar{\omega}^{\prime}+m_{m m} \bar{a}^{\prime}=C_{m m}^{0}+\sum_{k=1}^{\infty} C_{m m}^{k} \sin \left(k z \theta(t)+\psi_{m m}^{k}\right) \\
+ & \left\{n_{m m}^{0}+\sum_{k=1}^{\infty}\left[n_{m m}^{k} \sin \left(k z \theta(t)+\psi_{\omega^{\prime} m}^{k}\right)\right]\right\} \bar{\omega}^{\prime} \\
+ & \left\{m_{m m}^{0}+\sum_{k=1}^{\infty}\left[m_{m m}^{k} \sin \left(k z \theta(t)+\psi_{a^{\prime} m}^{k}\right)\right]\right\} \bar{a}^{\prime}
\end{aligned}
$$

The hydrodynamic coefficients of vertical-axis turbines are difficult to obtain directly because of dynamic stall and complex flow wakes. However, the time-history curves of $C_{F X}, C_{F Y}$ and $C_{M}$ can be obtained through numerical CFD calculations. Then, the least-squares method can be used to fit these curves to obtain the thrust, lateral force and yawing moment coefficients. The ANSYS-CFX software uses the finite-volume method based on finite elements to provide the conservation characteristics of the finite-volume method combined with the accuracy of the finite-element method. Moreover, CFX offers high accuracy in the simulation of rotating machinery because of its fully implicit coupling solver with multiple grids. An shear stress transpore (SST) model is the most suitable choice for simulating turbines. Least-squares fits can be easily achieved through programming and have been widely applied for curve fitting. Therefore, the methodology used in this paper relies on the least-squares method and CFD simulations to simulate various turbines with high simulation robustness.

\subsection{CFX simulation}

To monitor the stress state of a vertical-axis turbine during yawing, ANSYS-CFX was used to simulate the operating conditions of the turbine in the presence of waves. The two-bladed vertical-axis turbine used in this simulation model was developed by Harbin Engineering University (HEU). The diameter of the turbine is $0.8 \mathrm{~m}$, and the two blades have an NACA0018 airfoil profile; the chord length of each blade is $0.12 \mathrm{~m}$ (see Figure 1). A series of experiments was conducted on vertical-axis tidal turbines in the circulating water tunnel laboratory at HEU. The basic turbine parameters are provided above. The turbines were installed on a test platform, and the test platform was installed in the working segment $(8 \mathrm{~m} \times 1.7$ $\mathrm{m} \times 1.5 \mathrm{~m}$ ) of the circulating water tunnel. The hydrodynamic performance of the turbines was studied under various tunnel velocities, and the experiments were performed over a long period of time.

To calculate several turbine operating conditions, a 2D CFD model was used to represent the hydrodynamic performance of the vertical-axis turbine. The working principle of vertical-axis tidal current turbines is similar to that of vertical-axis wind turbines (VAWTs). Many relevant studies of VAWTs in the literature have shown that a 2D model is sufficient to reveal the factors that influence the performance of a VAWT and the flow physics in its surroundings. In 2014, Rosario simulated two different types of H-Darrieus wind turbines using 2D models and compared the calculations with experimental data. The results showed good agreement between the 2D numerical findings and the experimental data [19]. In 2016, the near and far wakes of a low-solidity VAWT with two straight blades were investigated by Lam ad Peng using 
both 2D and 3D CFD simulations. The comparisons showed that the wake results were similar between the 2D and 3D simulations. Furthermore, the 2D and 3D CFD models consistently predicted a rated $\lambda$ of 4.5 [20]. Other researchers have also used 2D CFD models to simulate the performance of VAWTs and have proven the accuracy of such 2D models, including Danao [21], Bedon [22], and Lim [23]. Therefore, a 2D CFD model can be considered a valid tool for investigating the performance of vertical-axis turbines.

The computational domain of the 2D grid was divided into the three sections shown in Figure 2: the rotation section (within the yawing section), the yawing section and the static section. The turbine's rotating and yawing motions were simulated simultaneously. The rotating and yawing motions were considered to be rotary motions, which were modelled using the slipping grid method. The grids did not undergo translations during rotation, and the quality of the grids remained unchanged; both of these characteristics are favourable for computational accuracy and speed. A yawing motion ( $\xi=A \sin \left(\omega_{Y} t\right)$ ) was applied to the rotation section and to the yawing section. Furthermore, rotary motion occurred concurrently around the centre of the rotation section, and the grids of the static section remained motionless.

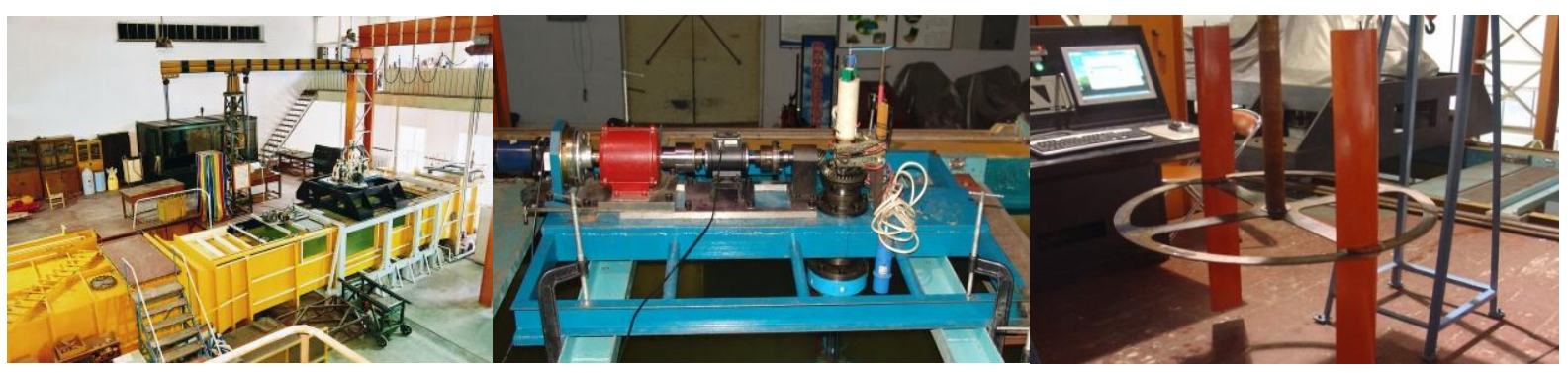

(a) Circulating water tunnel at HEU

(b) Test platform

(c) Two-bladed vertical-axis tidal turbine

Figure 1 Experimental facilities

Meshing is a very important aspect of numerical simulation technology; however, it is also one of the most time-consuming processes. The grid quality directly affects the accuracy and efficiency of a numerical calculation. The mesh structure of the entire computational domain was composed of structural grids, and the region closest to the blade was meshed with quadrilateral structured $\mathrm{O}$ grids, as shown in Figure 2. To select a suitable value for $\mathrm{Y}^{+}$, four different heights of the first layer of the turbine's surface mesh were defined: $\mathrm{Y}^{+}<1,1<\mathrm{Y}^{+}<10,10<\mathrm{Y}^{+}<20$, and $20<\mathrm{Y}^{+}<40$. Table 2 shows the detailed information about the different grid structures, and Figure 3 compares the tangential and normal force coefficients for the four meshes. The general trends for all four meshes are similar. The values for meshes 1 and 2 are nearly overlapping, and the values for meshes 3 and 4 differ only slightly. Therefore, mesh 2 was chosen to simulate the vertical-axis turbine because of its relatively high accuracy and lower computational cost.

The reference atmospheric pressure was established, and the inlet boundary was set to speed entry. The turbulence parameters and the speeds were set simultaneously, and pressure outlet boundary conditions with a relative pressure of 0 were used for the outlet. The sides of the computational domain were defined as free-slip walls, and the blade surfaces were defined as no-slip walls. In the rotation section, the rotational angular velocity and the yawing angular velocity were given; the rotation section and the yawing section were connected by an interface, as were the yawing section and the static section. The time period required for the blade to rotate $1^{\circ}$ was adopted as the time step for the entire simulation.

The turbulence model used in this paper is the SST model, which was proposed by Menter in 1994 [24]. The SST model accurately captures the extreme pressure and velocity gradients in the vicinity of turbine blades. Many researchers have proven that the SST model is the most suitable model for calculations concerning vertical-axis wind or water turbines, including Wang [25], Ponta [26], and Shiono et al. [27]. 


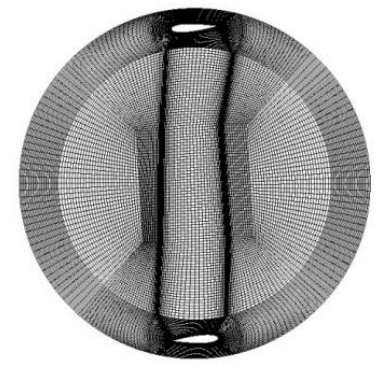

(a) Rotation section

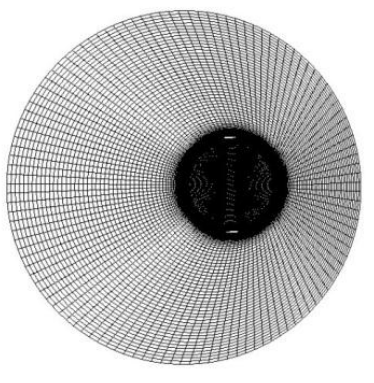

(b) Yawing section

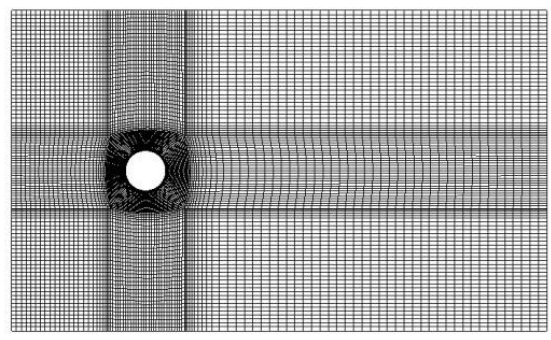

(c) Static section

Figure 2 Grid model

Table 2 Details of the different grid structures

\begin{tabular}{ccccc}
\hline Case & First layer thickness $(\mathrm{m})$ & Progression ratio & Number of rows & $\mathrm{Y}^{+}$ \\
\hline Mesh 1 & 0.00001 & 1.02 & 30 & $0.53-0.96$ \\
Mesh 2 & 0.00005 & 1.02 & 30 & $2.65-4.76$ \\
Mesh 3 & 0.0002 & 1.02 & 30 & $10.6-19.1$ \\
Mesh 4 & 0.0004 & 1.02 & 30 & $21.5-38.2$ \\
\hline
\end{tabular}

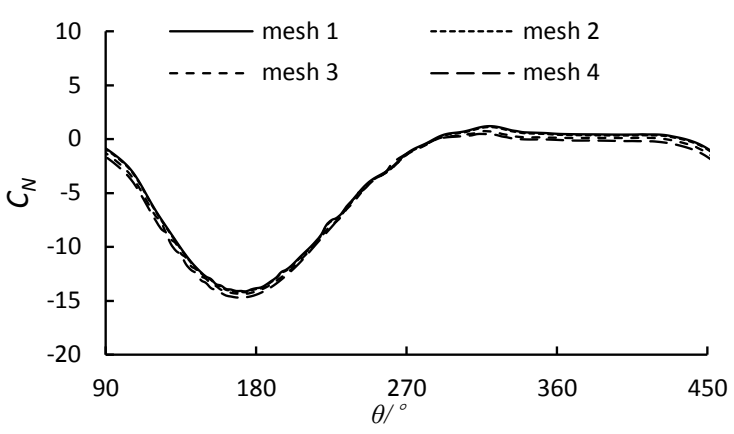

(a) Normal force coefficients

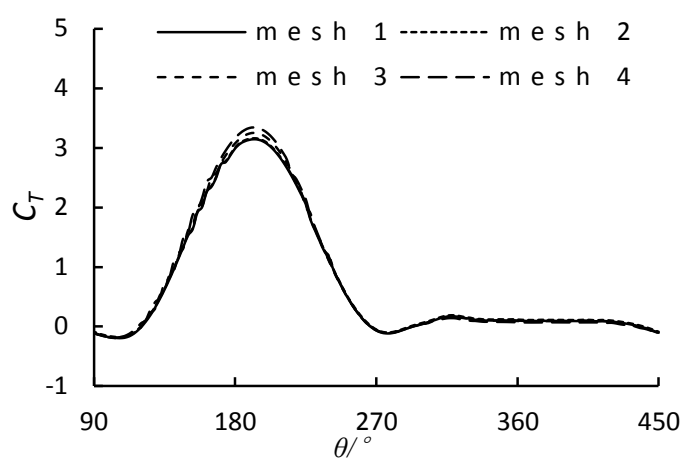

(b) Tangential force coefficients

Figure 3 Comparisons of the tangential and normal force coefficients for the different grids

The power coefficient was experimentally obtained; then, the experimental results were compared with the results of the 2D numerical calculations, the 2D correction and the 3D results, as illustrated in Figure 4. As seen from this comparison, the trends of the four sets of results are similar ( $\lambda=2.5$ is the optimal tip speed ratio), but the values obtained from the 2D calculations are obviously higher than the experimental results. In the experiments, 3D effects, arm effects, free surface effects and other factors negatively impacted the power output of the turbines and produced obvious differences between the turbine power coefficients found from the 2D calculations and the corresponding experimental values. In 2010, Ye $\mathrm{Li}$ studied the 3D effects and arm effects in the modelling of a vertical-axis tidal current turbine [28]. The results showed that 3D effects are significant when the ratio of the height of the turbine to its radius is less than 2.0 (i.e., $\mathrm{H} / \mathrm{R}<2.0$ ). $\mathrm{Li}$ compared the results with those for a 2D model and found that the maximum experimentally observed power coefficient was decreased by $19.5 \%$ compared with the modelled value (when $\mathrm{H} / \mathrm{R}=1.5$, which is the ratio for the turbine used in this study). Regarding arm effects, Li presented a relationship between TSR (the tip speed ratio) and the power coefficient for different arm profiles. When TSR $=2.5$, the power coefficient was decreased by 0.1 as a result of arm effects, corresponding to approximately $20 \%$ of the turbine power output. This gap continued to increase with increasing TSR. After the results of the 2D numerical calculations presented in this paper are corrected following $\mathrm{Li}$, the corrected values are approximately the same as the experimental results and the results of the 3D calculations, as shown in Figure 4. Therefore, with appropriate corrections, the 2D calculation method can be applied to effectively simulate tidal current turbines. 


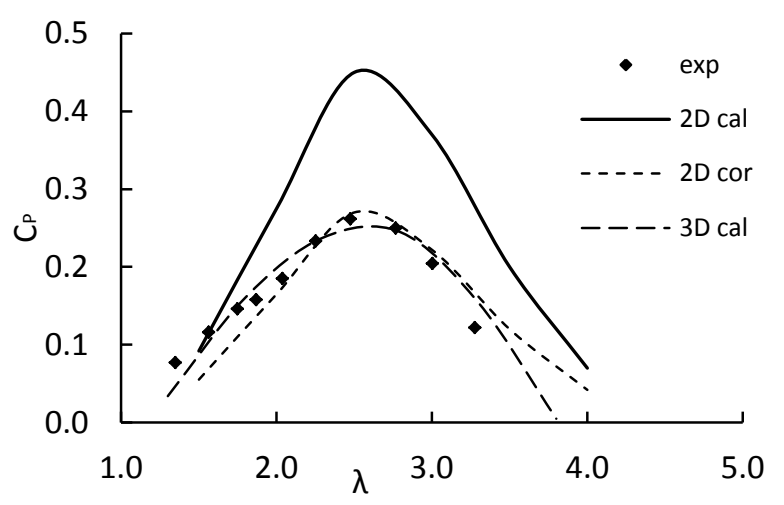

Figure 4 Comparison of power coefficients

To further confirm the accuracy of the 2D numerical method applied in this paper, the same method was used to simulate the classical vertical-axis turbine experiment (Texas Tech University, United States) [29]. In that 2D tunnel experiment, the tangential and normal force coefficients of the blades were measured, and the turbine used was similar to that used in this paper. The diameter of the turbine was $1.22 \mathrm{~m}$, the chord length was $0.0914 \mathrm{~m}$, and the two blades had a NACA symmetric airfoil profile (NACA0012). The same method was adopted to simulate this two-bladed vertical-axis turbine model.

The comparisons of the results for the tangential and normal force coefficients of the blade are as follows.

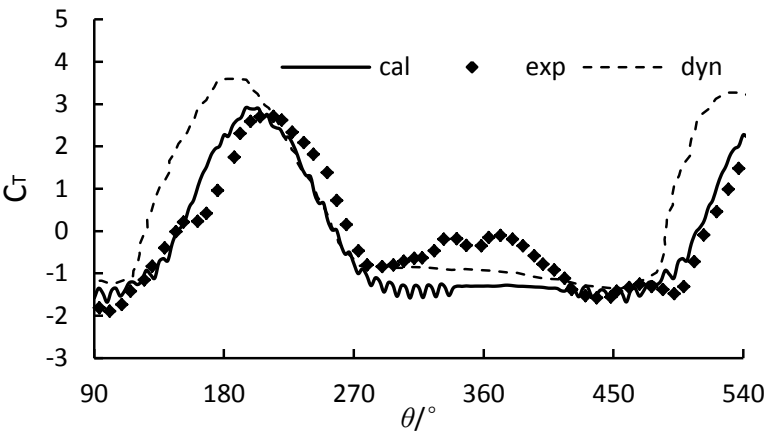

(a) Tangential force coefficients

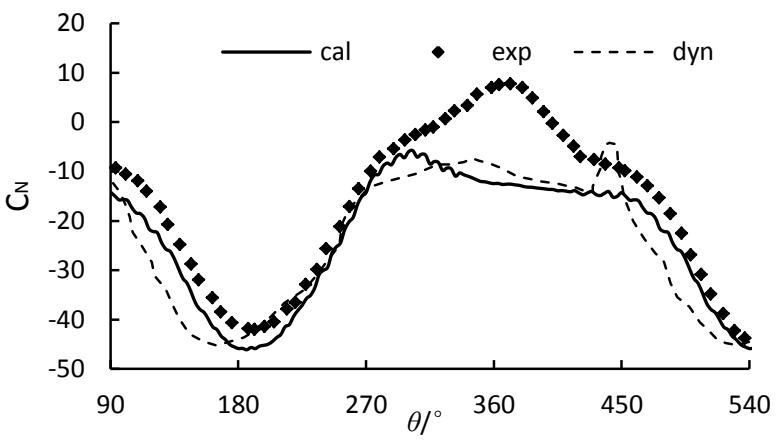

(b) Normal force coefficients

Figure 5 Comparisons between the calculated and experimental values

Figure 5 compares the simulated results (solid lines) from CFX, the experimental results (dotted lines) and the calculated results (dashed lines) from Texas Tech University's dynamic model. For a blade azimuth angle of between $270^{\circ}$ and $450^{\circ}$, the values from CFX and the dynamic model exhibited a goodness of fit and a larger difference with respect to the experimental results. The Texas Tech University results indicate that the hump observed when the blades are rotating downstream of the disk (at approximately $360^{\circ}$ ) may be partially due to misalignment errors in the blade mounting. Therefore, when the gap near blade azimuth angles of $360^{\circ}$ is disregarded, the comparison indicates satisfactory accuracy of the 2D CFX numerical method for vertical-axis tidal current turbines.

\section{Analysis of the results}

\subsection{Effects of yawing frequency on the turbine power coefficient}

The yawing frequency of the turbine is related to the wave frequency. To ensure accurate interpolation, yawing frequencies of $0.2,0.4,0.8,1.2,1.6$ and $2.0 \mathrm{rad} / \mathrm{s}$ were selected, with an incoming flow velocity of $V=1 \mathrm{~m} / \mathrm{s}$, an optimal turbine speed ratio of $\lambda=2.5$, and a yawing arm of $0.4 \mathrm{~m}(0.5 \mathrm{D})$. The graphs in Figure 6 show how the power coefficient varies under different operating conditions. Figure 6(a) illustrates the turbine operating environment in the case of a uniform flow as the turbine revolves around the fixed main axis. Figure 6(b) shows the yawing motion of the turbine as it revolves around the main axis with a given yawing frequency. The upper and lower envelopes in Figure 6(a) are approximately straight lines. By contrast, the turbine envelopes in the case of yawing motion, as illustrated in Figure 6(b), exhibit obvious cyclical 
oscillations. Figure 7(a) presents the differences in the power coefficients between non-yawing motion and yawing motion for different tip speed ratios. The comparison shows that the power coefficient under non-yawing motion is higher than that under yawing motion; the gap is particularly large for high tip speed ratios. Yawing motion changes the fluid speed distribution around the turbine, which changes the transient force and torque on the turbine and the transient values of the power coefficient. Figure 7(b) shows the differences in the blade tangential force coefficients between non-yawing motion and yawing motion. When the blade azimuth angle is between approximately $120^{\circ}$ and $200^{\circ}$, the $C_{f}$ for non-yawing motion is higher than that for yawing motion. Although the value of $C_{f}$ is higher under yawing motion from $290^{\circ}$ to $350^{\circ}$, this value is close to zero and therefore has a minimal effect on the turbine power output. As a result, the power coefficient under non-yawing motion is higher than that under yawing motion.

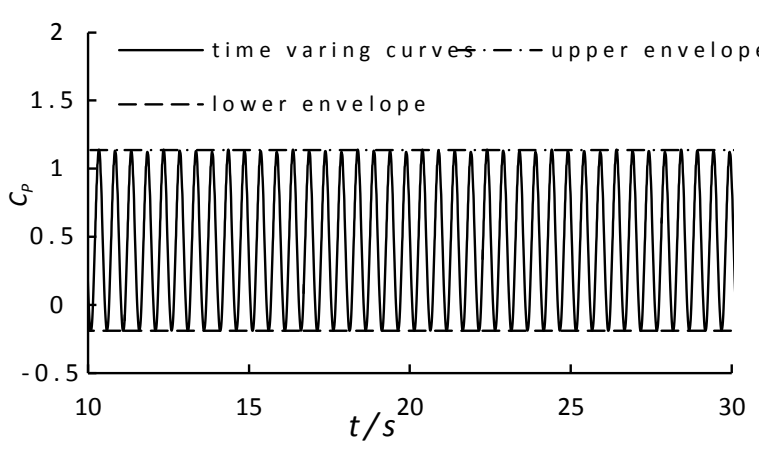

(a) Non-yawing motion

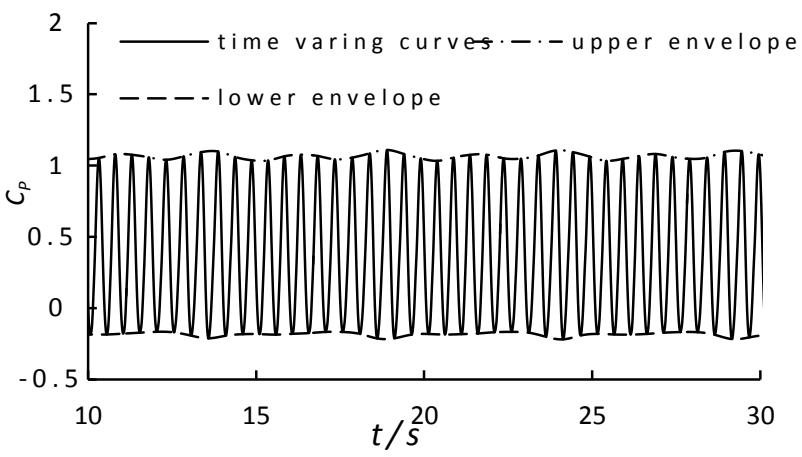

(b) Yawing motion

Figure 6 Time-varying curves for the power coefficient under different operating conditions

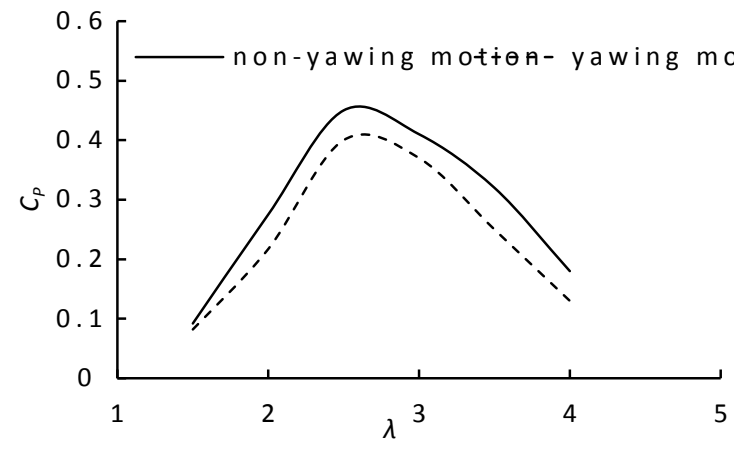

(a) Power coefficients

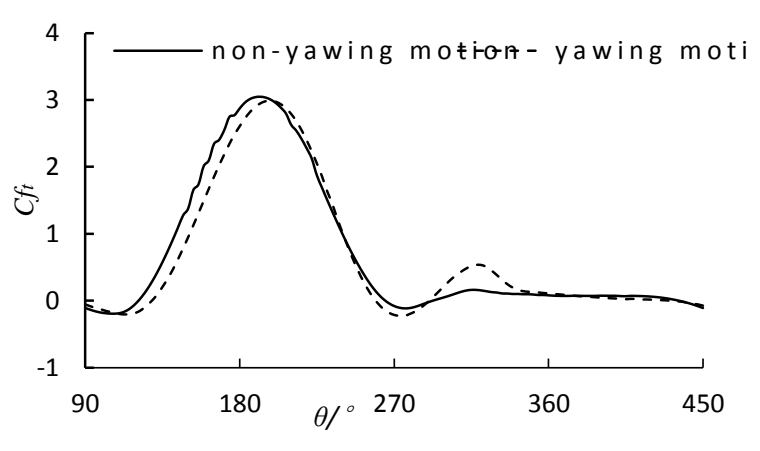

(b) Tangential force coefficients

Figure 7 Comparisons of the power coefficients and tangential force coefficients between non-yawing motion and yawing motion

In most cases, the average value of the power coefficient represents the average annual electricity production. Therefore, this paper presents the trends of the average value of the power coefficient under different yawing frequencies. Figure 8 shows that the power coefficient exhibits minimal variations with varying yawing frequencies at the optimal tip speed ratio ( $\lambda=2.5$ ) because the changes in the fluid speed distribution around the turbine are equivalent to the phenomenon in which the turbine is moving with a complicated speed profile. Occasionally, the speed distribution around the turbine under yawing motion is more complex than that under non-yawing motion; however, this has minimal impact on the output electricity. Ultimately, the annual energy production of such a turbine under yawing motion exhibits minimal variations.

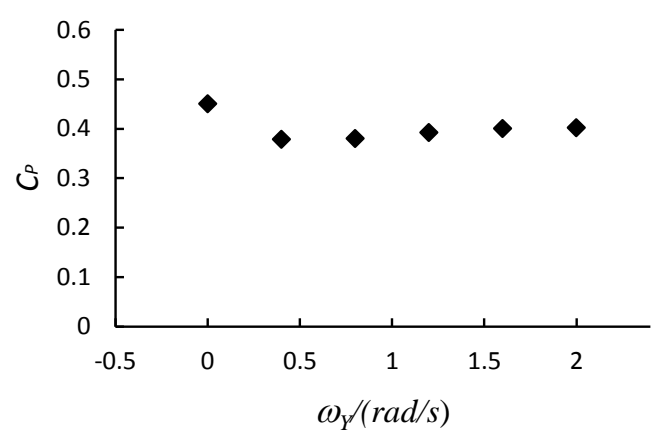

Figure 8 Average power coefficient values under different yawing frequencies 


\subsection{Effects of yawing frequency on the turbine loads}

The CFX software was used to calculate the time-varying curves for three coefficients $\left(C_{F X}, C_{F Y}, C_{M}\right)$, and the comparisons of these curves under different yawing frequencies are discussed below.

Figure 9(a) shows the time-varying lateral force coefficient curves for a turbine under non-yawing motion with an incoming flow speed of $1.0 \mathrm{~m} / \mathrm{s}$ and a speed ratio of 2.5 . The turbine loads regularly fluctuate in accordance with their rotational frequency, and the values for the wave crests and troughs remain stable over time, similar to the power coefficient. For the calculations presented in Figure 9(b), the yawing frequency was set to $1.2 \mathrm{rad} / \mathrm{s}$ and the amplitude was set to $A=10^{\circ}$. The incoming flow speed and speed ratio were the same as in the initial model. The time-varying load curves are affected by both the rotational and yawing frequencies. Compared with the yawing frequency, the rotational frequency $(6.25 \mathrm{rad} / \mathrm{s})$ is a high-frequency oscillation. Consequently, the turbine load oscillations can be divided into low-frequency oscillations (corresponding to the yawing frequency) and high-frequency oscillations (corresponding to the rotational frequency); the envelope stand fluctuates with load changes under yawing motion.

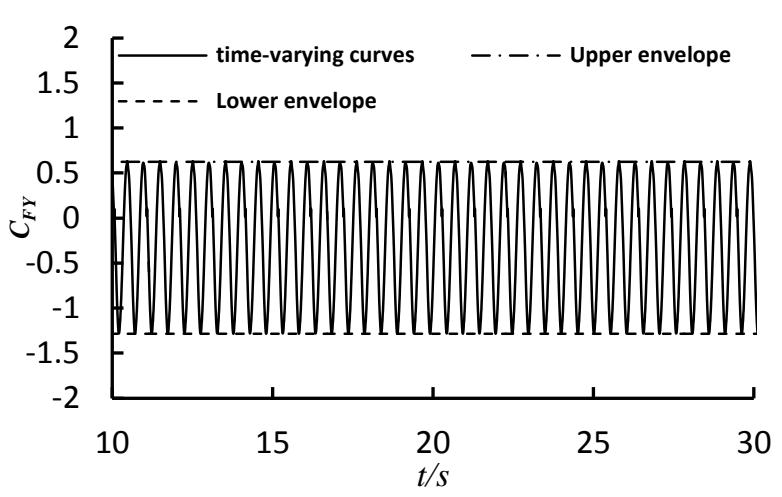

(a) Non-yawing motion

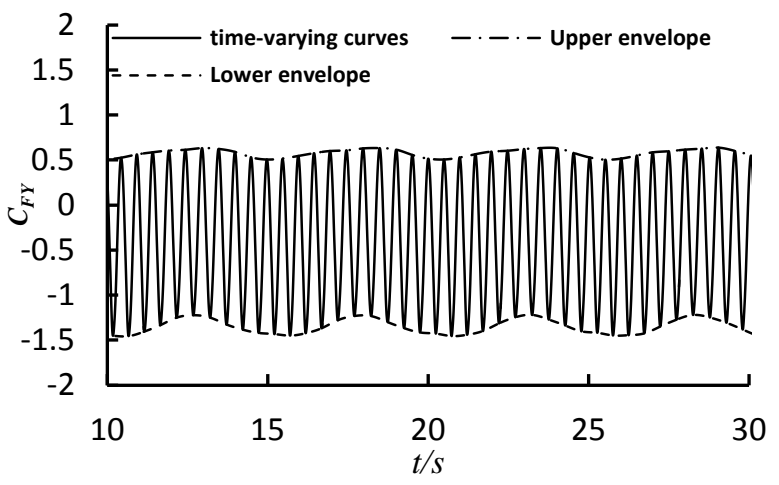

(b) Yawing motion

Figure 9 Load coefficients under yawing motion and non-yawing motion

Figures 10(a) and (b) show the upper and lower envelopes for the thrust coefficient curves, and Figures 10(c) and (d) show the lateral force coefficient envelopes. When waves cause the turbine to yaw, the load coefficient envelope curves fluctuate up and down with respect to the envelope curves under non-yawing motion. As the yawing frequency increases, the oscillation amplitudes of the envelope curves increase and the periods decrease. The turbine loads oscillate at high frequencies and with large amplitudes, adversely impacting the turbine intensity. A comparison of the upper and lower envelope curves for the load coefficients shows that the oscillation amplitudes of the upper envelope curves for the thrust coefficients are higher than those of the lower envelope curves and that the envelope curves for the lateral force coefficients fluctuate with large amplitudes. This difference is caused by the larger changes in the fluid speed distribution in the direction of the lateral force (Y direction) compared with those in the thrust direction ( $\mathrm{X}$ direction) under yawing motion.

The trends exhibited by the yawing moment coefficients at different yawing frequencies in Figures 10(e) and (f) are similar to those for the force coefficients described above. The oscillation amplitudes of the envelope curves increase with increasing yawing frequency, while their periods decrease. However, Table 3 shows that unlike the growing oscillation amplitudes, the average values remain relatively constant (at nearly -0.2) with increasing yawing frequency. 


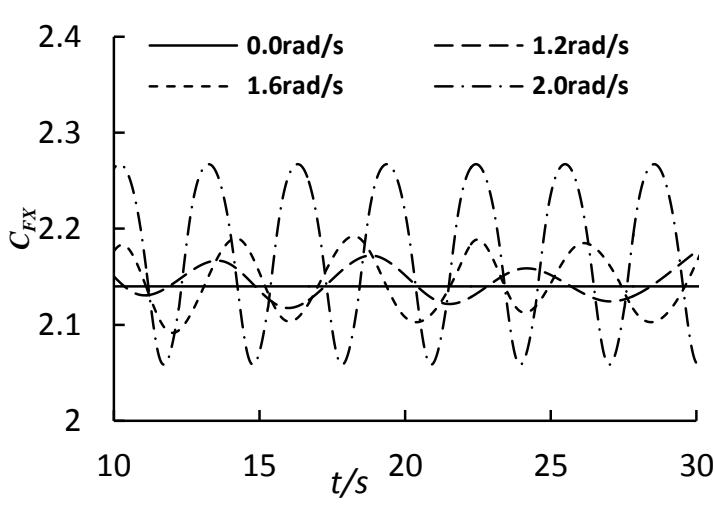

(a) Upper envelope curves for thrust coefficients

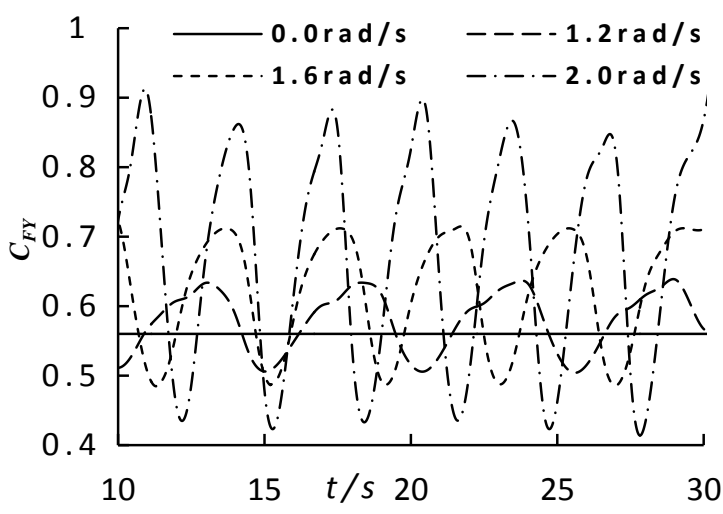

(c) Upper envelope curves for lateral force coefficients

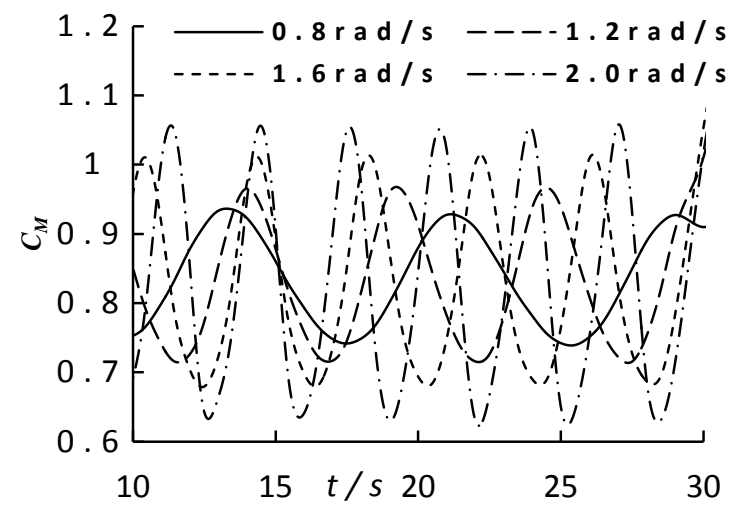

(e) Upper envelope curves for yawing moment coefficients

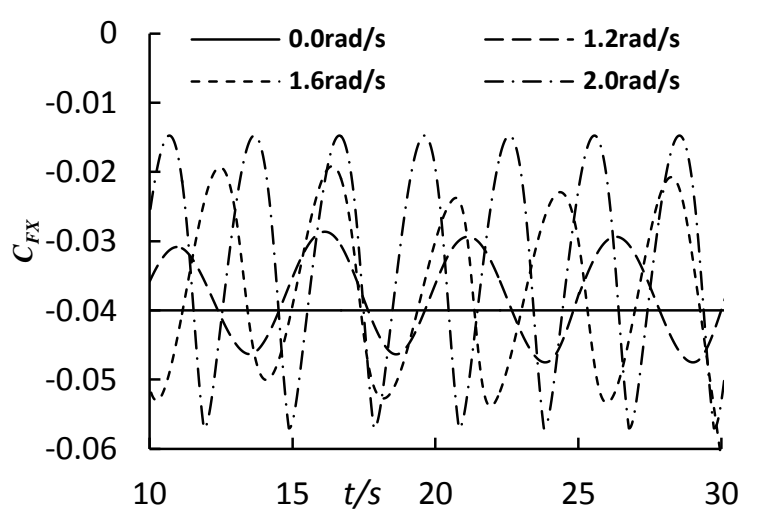

(b) Lower envelope curves for thrust coefficients

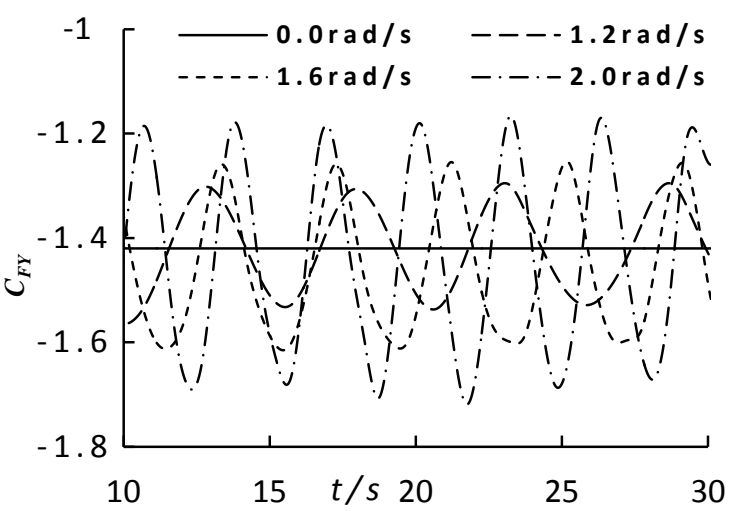

(d) Lower envelope curves for lateral force coefficients

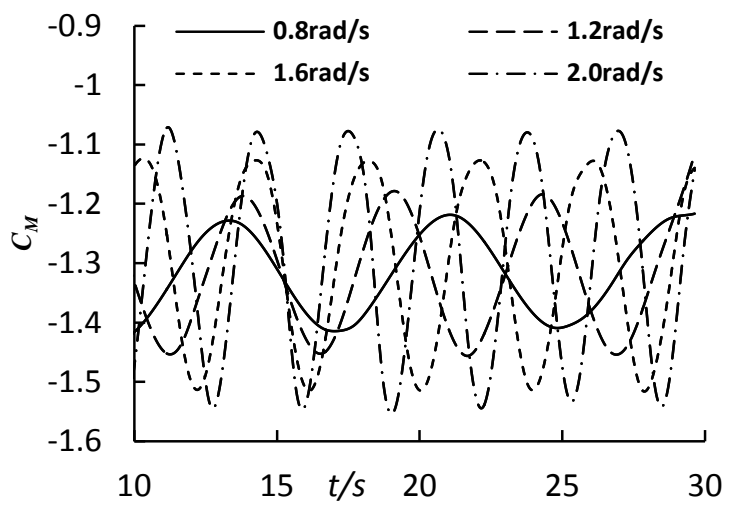

(f) Lower envelope curves for yawing moment coefficients

Figure 10 Envelope curves for the transient values of the force and moment coefficients at different yawing frequencies

The average values and oscillation amplitudes are defined as follows: $\bar{C}_{F X}=\int_{t_{1}}^{t_{1}^{2}} C_{F X} d t /\left(t_{2}-t_{1}\right), \bar{C}_{F Y}=\int_{t_{1}}^{t_{1}} C_{F Y} d t /\left(t_{2}-t_{1}\right)$, $\bar{C}_{M}=\int_{11}^{12} C_{M} d t /\left(t_{2}-t_{1}\right), \widehat{C}_{F X}=\left(C_{F X_{\max }}-C_{F X_{\min }}\right) / 2, \widehat{C}_{F Y}=\left(C_{F Y_{\max }}-C_{F Y_{\text {min }}}\right) / 2$, and $\widehat{C}_{M}=\left(C_{M_{\max }}-C_{M_{\min }}\right) / 2$. Table 3 shows the average values and oscillation amplitudes of the three load coefficients under the designated operating conditions (the incoming flow speed is 1.0 $\mathrm{m} / \mathrm{s}$, the speed ratio is 2.5 , and the amplitude is $A=10^{\circ}$ ). This table illustrates that the average values of the three load coefficients are minimally influenced by an increasing yawing frequency and that the oscillation amplitudes increase gradually. When the frequency is $1.6 \mathrm{rad} / \mathrm{s}$, the thrust coefficient oscillation amplitude can be up to $15 \%$ larger than that under non-yawing motion, and there is a $24 \%$ increase for the lateral force coefficient. The oscillation amplitude for the yawing moment coefficient under yawing motion (with a frequency of $1.6 \mathrm{rad} / \mathrm{s}$ ) is 1.26 , whereas that under non-yawing motion is zero. Therefore, the turbine structure should be strengthened for use under high-frequency wave conditions.

Table 3 Comparison of average values and oscillation amplitudes

\begin{tabular}{ccccccc}
\hline$\omega_{Y}$ & $\overline{C_{F X}}$ & $C_{F X}$ & $\overline{C_{F Y}}$ & $C_{F Y}$ & $\overline{C_{M}}$ & $C_{M}$ \\
\hline 0 & 1.001 & 0.983 & -0.350 & 0.937 & 0 & 0
\end{tabular}




\begin{tabular}{lllllll}
0.4 & 1.006 & 1.034 & -0.356 & 0.975 & -0.201 & 1.12 \\
0.8 & 1.014 & 1.038 & -0.352 & 1.001 & -0.197 & 1.18 \\
1.2 & 1.034 & 1.113 & -0.362 & 1.105 & -0.210 & 1.21 \\
1.6 & 1.038 & 1.129 & -0.363 & 1.164 & -0.204 & 1.26 \\
\hline
\end{tabular}

\subsection{Pressure analysis of blades under different yawing frequencies}

To study the mechanisms governing the load fluctuations of turbines under yawing motion, the pressure contours of the blades under different yawing frequencies are analysed. Figure 11 shows the blade pressure contours under four yawing frequencies $\left(\omega_{Y}=0.0 \mathrm{rad} / \mathrm{s}, 0.8 \mathrm{rad} / \mathrm{s}, 1.2 \mathrm{rad} / \mathrm{s}\right.$, and $\left.1.6 \mathrm{rad} / \mathrm{s}\right)$. To obtain accurate analysis results, the four blades were selected to have the same blade azimuth angle and yawing position (namely, $\xi=A^{*} \sin \left(\omega_{Y} t\right)=0^{\circ}$ ).

Figure 11(a) shows the pressure distribution of the blades under non-yawing motion. The zone boundary of the pressure contour is smoother and smaller than those of the pressure contours of the blades under the three cases of yawing motion. Under the yawing motion of the turbine, the blades generate another linear velocity in addition to the linear velocity induced by the turbine's own rotation. Under the combined effect of these two linear velocities, the resultant velocity under yawing motion is more complex and fluctuates. A comparison of Figures 11(a) and (d) reveals that the high-pressure zone in Figure 11(d) is larger and that the zone boundary is less smooth than the boundary presented in Figure 11(a). Therefore, blades subjected to high frequencies experience higher pressures, and more severe oscillations arise in the blade loads, thereby posing a critical challenge to the structural strength and fatigue strength of turbines operating under complex marine conditions.

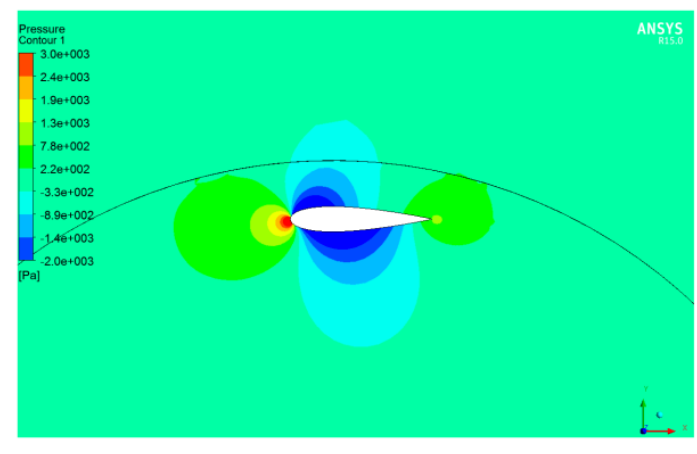

(a) $0.0 \mathrm{rad} / \mathrm{s}$

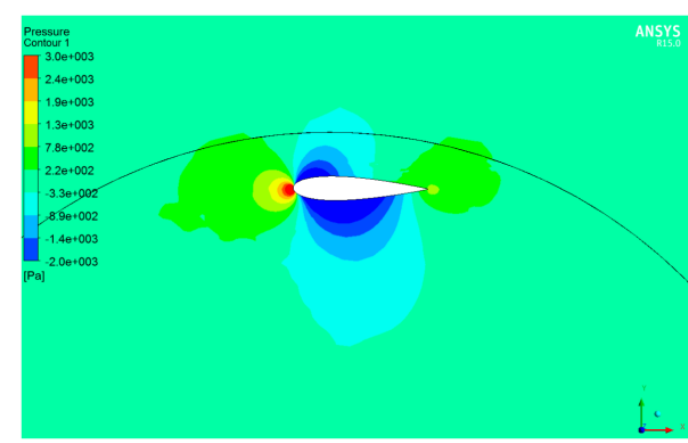

(c) $1.2 \mathrm{rad} / \mathrm{s}$

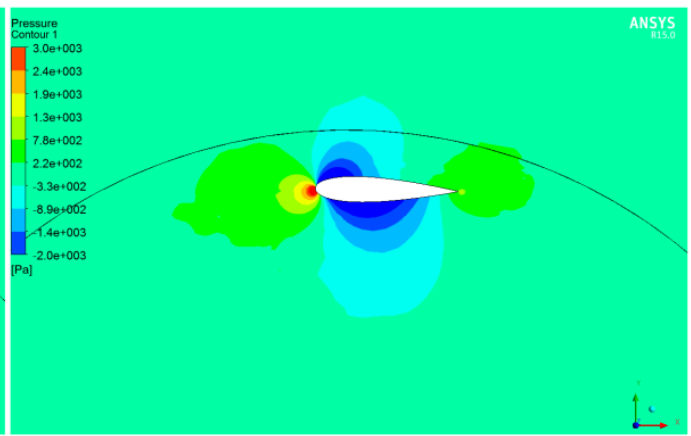

(b) $0.8 \mathrm{rad} / \mathrm{s}$

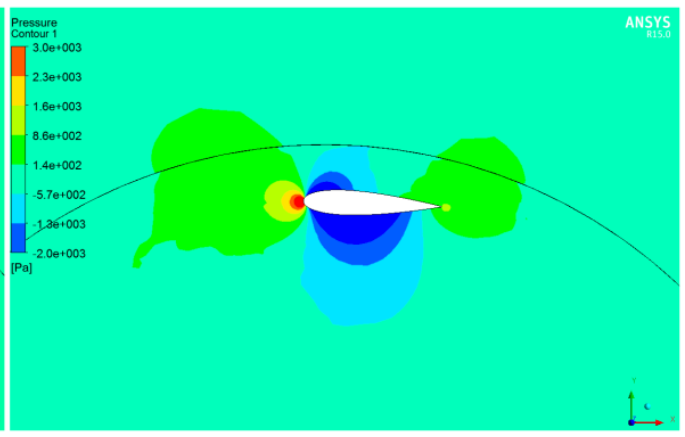

(d) $1.6 \mathrm{rad} / \mathrm{s}$

Figure 11 Comparison of the pressure contours of the blades under different yawing frequencies

Figures 12 (a)-(d) show the vortex distributions of the turbine under different yawing frequencies. Four yawing frequencies $\left(\omega_{Y}=0.0 \mathrm{rad} / \mathrm{s}, 0.8 \mathrm{rad} / \mathrm{s}, 1.2 \mathrm{rad} / \mathrm{s}\right.$, and $\left.1.6 \mathrm{rad} / \mathrm{s}\right)$ were selected to analyse and compare the different vortex distributions. Figure 12(a) shows the vortex distribution of the turbine under the yawing frequency of $\omega_{Y}=0.0 \mathrm{rad} / \mathrm{s}$; in this case, the turbine rotates only in a uniform flow, the vortex generated from the blade tails is predominantly symmetric, and the vortex strength slowly decreases throughout the spreading process. Figures 12(b), (c) and (d) show the turbine vortex distributions under yawing motion. Here, the turbine not only rotates around a fixed axis but also yaws because of 
the complex marine conditions. The vortex distributions are clearly asymmetric, and more vortices are generated and oscillate with the yawing motion. At high frequencies, the yawing motion causes wakes, similar to a Karman vortex street. As the yawing frequency increases, the trailing vortices oscillate in a progressively higher frequency range and gradually disappear. The generation of vortices under yawing motion removes available power from the turbines, decreasing the power coefficient compared with that achieved when the turbine is operating under non-yawing motion. The vortices also oscillate with the yawing period, similar to the cyclical oscillations experienced by the loads and yawing moments, as reported above. Furthermore, the yawing motion more obviously influences the water flow farther downstream.

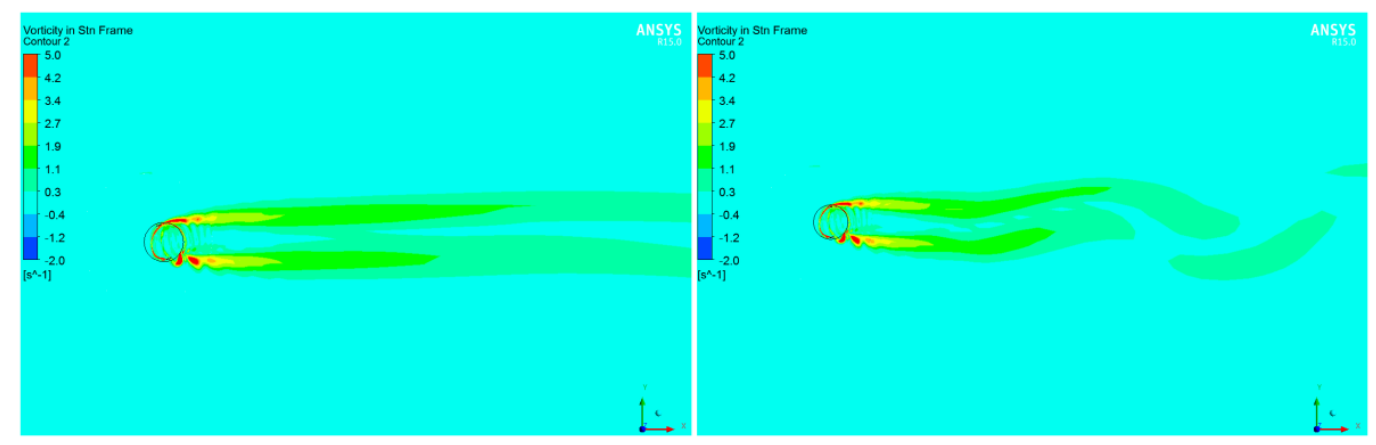

(a) $0.0 \mathrm{rad} / \mathrm{s}$

(b) $0.8 \mathrm{rad} / \mathrm{s}$

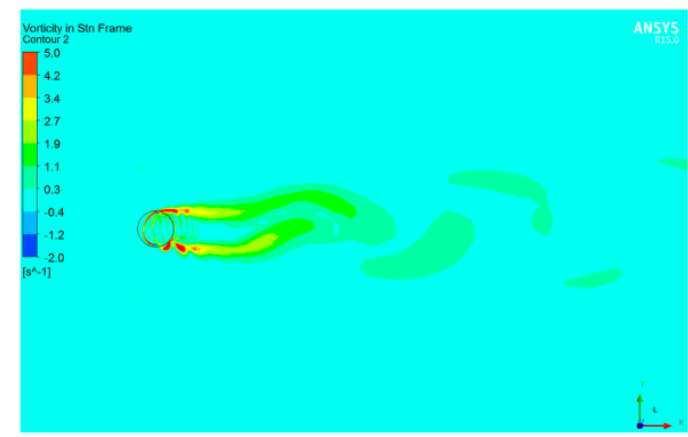

(c) $1.2 \mathrm{rad} / \mathrm{s}$

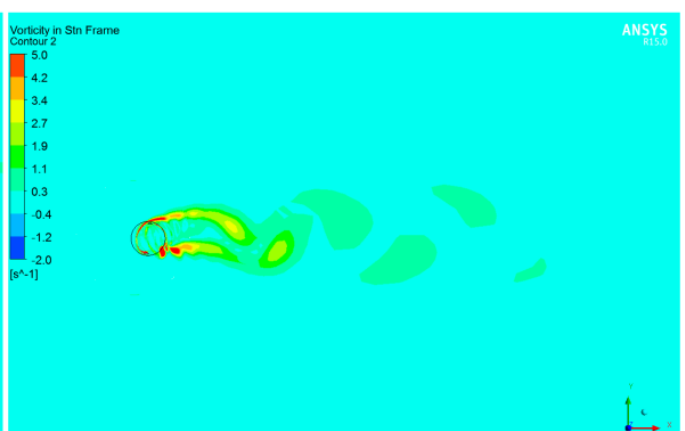

(d) $1.6 \mathrm{rad} / \mathrm{s}$

Figure 12 Comparison of vortex distributions under different yawing frequencies

\section{Fitting analysis of the vertical-axis turbine under different yawing frequencies}

The time-varying curves of the thrust coefficient, the lateral force coefficient and the yawing moment coefficient under yawing motion can be described by means of CFD simulations. The damping and added mass coefficients can then be obtained using the least-squares method, which can be applied to fit the three coefficients using formulas (18), (19) and (20). Figure 13 shows the comparison between the CFD curves and the fitted results under the operating conditions given by $V=1.0 \mathrm{~m} / \mathrm{s}, \lambda=2.5, \omega_{Y}=1.2 \mathrm{rad} / \mathrm{s}$ and $\mathrm{A}=10^{\circ}$, in which the fitted results are found to be satisfactory.

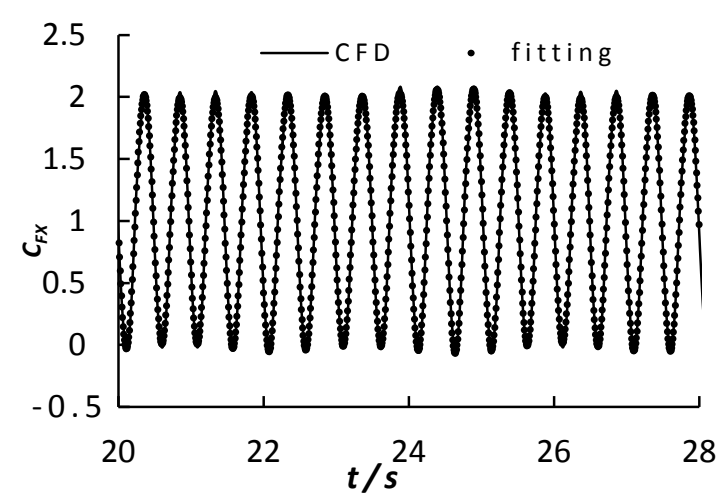

(a) Thrust coefficient

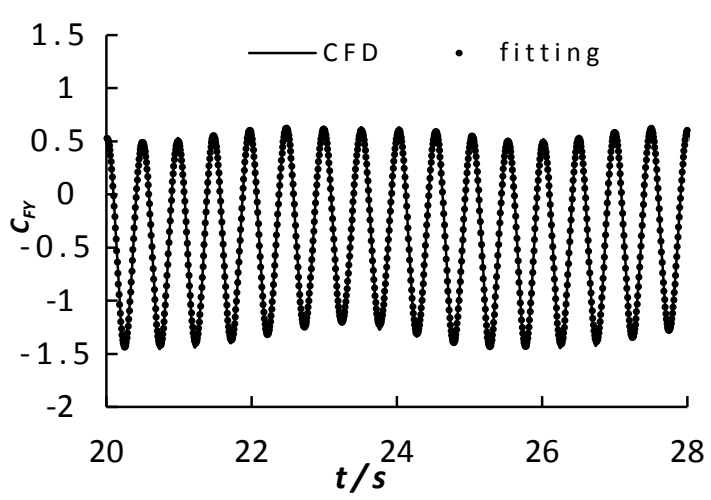

(b) Lateral force coefficient 


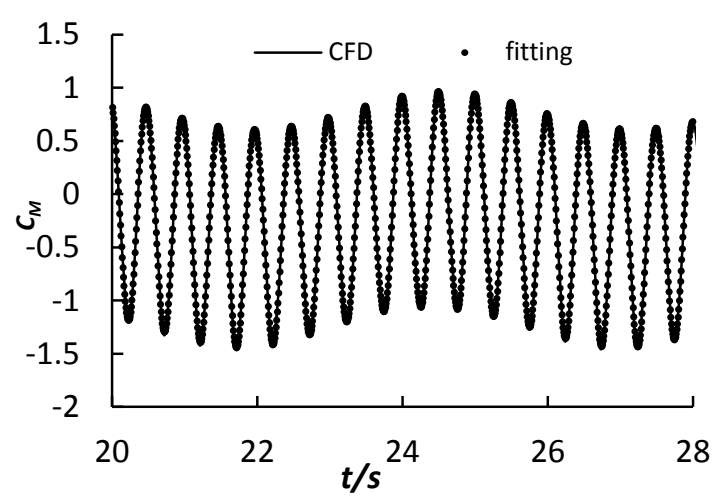

(c) Yawing moment coefficient

Figure 13 Comparison between CFD curves and the corresponding fitted results

\subsection{Effects of yawing frequency on turbine thrust}

Table 4 shows the coefficients for the constant and first-order terms of $\mathrm{C}_{\mathrm{FX}}$, which were obtained by using the least-squares method to fit the time-varying curves of the thrust coefficients under different yawing frequencies.

Table $4 C_{F X}$ expansion coefficient table

\begin{tabular}{ccccccccccc}
\hline$\omega_{Y}$ & $C_{x x}^{0}$ & $C_{x x}^{1}$ & $\psi_{x x}^{1}$ & $n_{x x}^{0}$ & $n_{x x}^{1}$ & $\psi_{\omega^{\prime} x}^{1}$ & $m_{x x}^{0}$ & $m_{x x}^{1}$ & $\psi_{a^{\prime} x}^{1}$ \\
\hline 0.2 & 1.00 & 0.98 & -79.7 & -0.212 & 0.813 & 17.8 & 1.051 & 82.167 & -169.8 \\
0.4 & 1.00 & 0.98 & -79.8 & -0.203 & 0.793 & 10.7 & 0.996 & 75.586 & -169.5 \\
0.8 & 1.01 & 0.97 & -79.7 & -0.196 & 0.777 & 19.6 & 0.387 & 18.593 & -168.8 \\
1.2 & 1.02 & 1.00 & -79.4 & -0.158 & 0.722 & 20.9 & 0.366 & 7.877 & -166.5 \\
1.6 & 1.04 & 1.03 & -79.0 & -0.136 & 0.696 & 16.6 & 0.353 & 4.083 & -163.0 \\
2.0 & 1.03 & 1.02 & -78.9 & -0.066 & 0.664 & 7.14 & 0.318 & 2.385 & -157.5 \\
\hline
\end{tabular}

The homogeneous hydrodynamic expansion coefficients $\left(c_{x x}^{0}\right.$ and $\left.c_{x x}^{1}\right)$ for the vertical-axis turbine thrust coefficient are independent of the acceleration and velocity of the yawing motion. As the yawing frequency increases, the constant and first-order terms of the homogeneous hydrodynamic coefficient remain stable. However, the absolute values of the corresponding terms of the damping coefficient and the added mass coefficient decrease; furthermore, the influences of the damping and added mass coefficients on the yawing motion of the turbine are of the same order. Therefore, the yawing frequency has no influence on the homogeneous hydrodynamic coefficient; the added mass and damping terms are the two main factors that impose a thrust on a vertical-axis turbine under yawing motion.

\subsection{Effects of yawing frequency on turbine lateral force}

Table 5 shows the coefficients for the constant and first-order terms of $\mathrm{C}_{\mathrm{FY}}$, which were obtained by using the least-squares method to fit the time-varying curves of the lateral force coefficients under different yawing frequencies.

Table $5 C_{F Y}$ expansion coefficient table

\begin{tabular}{cccccccccc}
\hline$\omega_{Y}$ & $C_{y y}^{0}$ & $C_{y y}^{1}$ & $\psi_{y y}^{1}$ & $n_{y y}^{0}$ & $n_{y y}^{1}$ & $\psi_{\omega^{\prime} y}^{1}$ & $m_{y y}^{0}$ & $m_{y y}^{1}$ & $\psi_{a^{\prime} y}^{1}$ \\
\hline 0.2 & -0.364 & 0.864 & 175.9 & -0.746 & 0.639 & -127.6 & -0.486 & 75.28 & 86.2 \\
0.4 & -0.360 & 0.878 & 175.6 & -0.776 & 0.628 & -121.1 & -0.526 & 69.21 & 86.0 \\
0.8 & -0.364 & 0.893 & 175.8 & -0.796 & 0.647 & -114.7 & -0.566 & 17.14 & 86.2 \\
1.2 & -0.364 & 0.928 & 176.1 & -0.837 & 0.725 & -118.7 & -0.754 & 7.47 & 87.9 \\
1.6 & -0.364 & 0.981 & 176.5 & -0.956 & 0.841 & -107.2 & -0.842 & 4.07 & 89.0 \\
2.0 & -0.363 & 1.020 & 176.3 & -1.251 & 1.130 & -100.8 & -0.889 & 2.57 & 88.1 \\
\hline
\end{tabular}

Table 5 shows that the constant terms (absolute values) of the damping and added mass coefficients and the first-order 
terms (absolute value) of the homogeneous hydrodynamic coefficient and the damping coefficient all increase slightly with increasing frequency. However, the first-order term of the added mass coefficient decreases with increasing frequency, while the constant term (absolute value) of the homogeneous hydrodynamic coefficient remains approximately constant. When the yawing frequency changes, the acceleration and velocity of the yawing motion on the $\mathrm{Y}$ axis change correspondingly. These changes lead to variations in the three terms of the lateral force coefficient under different yawing frequencies. The reported values of $\psi_{y y}^{1}, \psi_{\omega^{\prime} y}^{1}$ and $\psi_{a^{\prime} y}^{1}$ reveal that these three terms vary periodically with the blade azimuth angle $\theta$.

\subsection{Effects of yawing frequency on turbine yawing moment}

Table 6 shows the coefficients for the constant and first-order terms of $\mathrm{C}_{\mathrm{M}}$, which were obtained by using the least-squares method to fit the time-varying curves of the yawing moment coefficients under different yawing frequencies.

Table $6 C_{M}$ expansion coefficient table

\begin{tabular}{ccccccccccc}
\hline$\omega_{Y}$ & $C_{m m}^{0}$ & $C_{m m}^{1}$ & $\psi_{m m}^{1}$ & $n_{m m}^{0}$ & $n_{m m}^{1}$ & $\psi_{\omega^{\prime} m}^{1}$ & $m_{m m}^{0}$ & $m_{m m}^{1}$ & $\psi_{a^{\prime} m}^{1}$ \\
\hline 0.2 & -0.201 & 0.958 & -170.3 & -0.834 & 0.518 & -75.6 & 45.89 & 43.17 & 101.5 \\
0.4 & -0.200 & 0.966 & -170.0 & -0.856 & 0.594 & -70.5 & 38.70 & 36.67 & 99.8 \\
0.8 & -0.201 & 0.979 & -169.8 & -0.864 & 0.644 & -99.8 & 9.35 & 8.86 & 102.3 \\
1.2 & -0.205 & 1.008 & -169.3 & -0.870 & 0.661 & -101.7 & 3.79 & 3.60 & 101.6 \\
1.6 & -0.204 & 1.039 & -168.8 & -0.962 & 0.745 & -92.0 & 1.80 & 1.71 & 103.9 \\
2.0 & -0.202 & 1.059 & -168.7 & -1.220 & 1.057 & -88.6 & 0.93 & 0.88 & 98.5 \\
\hline
\end{tabular}

Table 6 shows that with an increasing yawing frequency, the values of $C_{m m}^{0}$ and $C_{m}^{1}$ remain fixed at -0.2 and 1.0, respectively, which indicates that the homogeneous hydrodynamic expansion coefficient is not affected by the yawing frequency. As the yawing frequency increases, the constant term (absolute value) and first-order term (absolute value) of the damping coefficient slowly increase, and the corresponding terms (absolute values) of the added mass coefficient rapidly decrease. A change in the yawing frequency causes the angular velocity and angular acceleration to change; therefore, the variation in $C_{M}$ with the variation in the yawing frequency arises from changes in $C_{m m}^{0}, n_{m m}^{0}$ and $m_{m m}^{0}$. Additionally, the variation in $C_{M}$ with the variation in the impeller rotation arises from changes in $C_{m m}^{1}, n_{m m}^{1}$ and $m_{m m}^{1}$.

\subsection{Application of turbine simulations}

The above three tables show the expansion coefficients for the thrust, lateral force and yawing moment under different yawing frequencies. From these results, we can obtain a series of expansion coefficients for any yawing frequency (0-2.0 $\mathrm{rad} / \mathrm{s}$ ) through interpolation. Then, these expansion coefficients can be applied to a floating turbine platform to simulate the motion responses of the platform. Therefore, we can obtain the motion response under any yawing frequency for a floating vertical-axis tidal current turbine station, which will be a key focus of our future research.

Figure 14 shows the flow diagram for the method used to study the hydrodynamic performance of a floating vertical-axis tidal current turbine power station. CFD simulations and the least-squares method are used to obtain the added mass and damping coefficients for a turbine under yawing motion, which is the major research focus of this paper. Then, the hydrodynamic coefficients of the platform and mooring system are introduced to analyse the time-domain and frequency-domain motion responses. Therefore, our future work will be to analyse the response of the entire floating turbine power station in the time and frequency domains. 


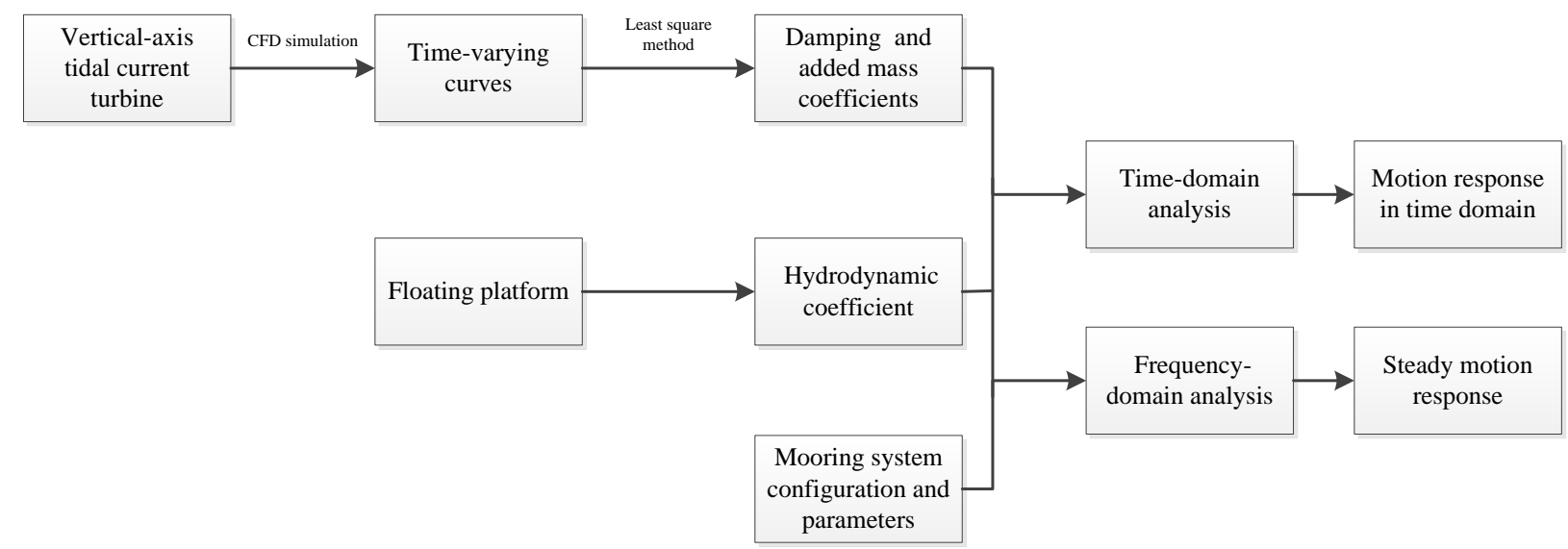

Figure 14 Method of studying a floating vertical-axis tidal current turbine power station

\section{Conclusions}

The hydrodynamic performance of a yawing vertical-axis turbine in a constant-inflow environment was obtained using $\mathrm{CFX}$, and the least-squares fitting method was applied to obtain the values of the components of $C_{F X} C_{F Y}$ and $C_{M}$. This study demonstrates the following:

1. Yawing motion cannot be ignored in the design of a floating vertical-axis tidal current power station. The average values of the thrust coefficient, lateral force coefficient and yawing moment coefficient are only marginally affected by the yawing motion of the turbine; however, the yawing frequency is positively correlated with the oscillation amplitudes of these three coefficients. In particular, at high frequencies, the lateral force coefficient exhibits an oscillation amplitude that is up to $24 \%$ larger than that under non-yawing motion. Therefore, turbines subjected to high-frequency waves require greater structural strength to extend their fatigue life.

2. The power coefficient under non-yawing motion is higher than that under yawing motion as a result of the lower blade tangential force coefficient, especially at higher tip speed ratios. Therefore, yawing motion can take a heavy toll on the annual electricity output of a power station.

3. Analyses of the loads and yawing moment of the turbine using the least-squares method reveal that the added mass and damping coefficients are the two main factors that impose loads and moments on a vertical-axis turbine under yawing motion.

4. The constant and first-order terms of the added mass components of the thrust, lateral force and yawing moment coefficients rapidly decrease with an increasing yawing frequency. The trends exhibited by the constant and first-order terms of the damping component of the thrust coefficient are opposite to those of the lateral force and yawing moment coefficients. This study provides a firm foundation for research regarding the motion response of floating support structures for offshore floating tidal current power stations.

\section{Acknowledgements}

This research work was financially supported by the National Science Foundation of China (No.51209060, No.51409065 and No.11572094). It is also supported by the Harbin Special Study Fund for technological innovation (No.2014RFQXJ017 and No.2015RQQXJ014) and by the National Science Foundation of Heilongjiang Province (E2016021).

\section{References}

[1] S. Wang, P. Yuan, D. Li, Y. Jiao, An overview of ocean renewable energy in china, Renewable Sustain. Energ. Rev. 15 (2011) 91-111. doi: 10.1016/j.rser.2010.09.040.

[2] F. O Rourke, F. Boyle, A. Reynolds, Tidal energy update 2009, Appl. Energ. 87 (2010) 398-409. doi: 10.1016/j.apenergy.2009.08.014. 
[3] Y. Ma, L. Zhang, L. Ma, Z. Chen, Development status and development trend of vertical axis turbine-type tidal current energy power generation device, Sci. Technol. Rev. 12 (2012) 71-5.

[4] W.M.J. Batten, A.S. Bahaj, A.F. Molland, J.R. Chaplin, The prediction of the hydrodynamic performance of marine current turbines, Renewable Energ. 33 (2008) 1085-96. doi: 10.1016/j.renene.2007.05.043.

[5] Y. Li, S.M. Çalişal, Numerical analysis of the characteristics of vertical axis tidal current turbines, Renewable Energ. 35 (2010) 435-42. doi: 10.1016/j.renene.2009.05.024.

[6] F. Jing, N. Mehmood, L. Zhang, G. Xiao, Optimal selection of floating platform for tidal current power station. Research Journal of Applied Sciences, Engineering and Technology 6 (2013) 1116-1121.

[7] R.W. Yeung, Added mass and damping of a vertical cylinder in finite-depth waters, Appl. Ocean Res. 3 (1981) 119-33. doi: 10.1016/0141-1187(81)90101-2.

[8] D.B.S. Lopes, A.J.N.A. Sarmento, Hydrodynamic coefficients of a submerged pulsating sphere in finite depth, Ocean Eng. 29 (2002) 1391-8. doi: 10.1016/S0029-8018(01)00081-6.

[9] S. Krenk, Time-domain analysis of frequency dependent inertial wave forces on cylinders, Comput. Struct. 126 (2013) 184-92. doi: 10.1016/j.compstruc.2012.09.004.

[10] Y. Li, M. Lin, Hydrodynamic coefficients induced by waves and currents for submerged circular cylinder, Procedia Eng. 4 (2010) 253-61. doi: 10.1016/j.proeng.2010.08.029.

[11] P. Hu, G.X. Wu, Q.W. Ma, Numerical simulation of nonlinear wave radiation by a moving vertical cylinder, Ocean Eng. 29 (2002) 1733-50. doi: 10.1016/S0029-8018(02)00002-1.

[12] C.L. Pavon, A.S. Iglesias, Hydrodynamic coefficients and pressure loads on heave plates for semi-submersible floating offshore wind turbines: A comparative analysis using large scale models, Renewable Energ. 81 (2015) 864-81.

[13] P.W. Galloway, L.E. Myers, A.S. Bahaj, Studies of a scale tidal turbine in close proximity to waves. Proc. Third International Conference and Exhibition on Ocean Energy, Bilbao, Spain, 6-8 Oct. 2010.

[14] I.A. Milne, A.H. Day, R.N. Sharma, R.G.J. Flay, Blade loads on tidal turbines in planar oscillatory flow, Ocean Eng. 60 (2013) 163-74. doi: 10.1016/j.oceaneng.2012.12.027.

[15] L. Zhang, S. Wang, Q. Sheng, F. Jing, Y. Ma, The effects of surge motion of the floating platform on hydrodynamics performance of horizontal-axis tidal current turbine, Renewable Energ. 74 (2015) 796-802. doi: 10.1016/j.renene.2014.09.002.

[16] F. Scheurich, R.E. Brown, Vertical-Axis wind turbines in oblique flow: sensitivity to rotor geometry, Proceedings of the European Wind Energy Conference and Exhibition 2011, Brussels, Belgium, (2011).

[17] A. Orlandi, M. Collu, S. Zanforlin, A. Shires, 3D URANS analysis of a vertical axis wind turbine in skewed flows, J. Wind Eng. Ind. Aerodynamics 147 (2015) 77-84. doi: 10.1016/j.jweia.2015.09.010.

[18] G. Bedon, S. De Betta, E. Benini, A computational assessment of the aerodynamic performance of a tilted Darrieus wind turbine, J. Wind Eng. Ind. Aerodynamics 145 (2015) 263-9. doi: 10.1016/j.jweia.2015.07.005.

[19] R. Lanzafame, S. Mauro, M. Messina, 2D CFD modeling of H-Darrieus wind turbines using a transition turbulence model, Energ. Procedia 45 (2014) 131-40. doi: 10.1016/j.egypro.2014.01.015.

[20] H.F. Lam, H.Y. Peng, Study of wake characteristics of a vertical axis wind turbine by two- and three-dimensional computational fluid dynamics simulations, Renewable Energ. 90 (2016) 386-98. doi: 10.1016/j.renene.2016.01.011. 
[21] A.D. Louis, H. Robert, Effects on the performance of vertical Axis wind turbines with unsteady wind inflow: A Numerical Study. 50th AIAA Aerospace Sciences Meeting Including the New Horizons Forum and Aerospace Exposition, Nashville, Tenn., United States. January 9-12, 2012.

[22] G. Bedon, S. De Betta, E. Benini, Performance-optimized airfoil for Darrieus wind turbines, Renewable Energ. 94 (2016) 328-40. doi: 10.1016/j.renene.2016.03.071.

[23] Y.C. Lim, W.T. Chong, F.B. Hsiao, Performance investigation and optimization of a vertical Axis wind turbine with the Omni-direction-guide-vane, Procedia Eng. 67 (2013) 59-69. doi: 10.1016/j.proeng.2013.12.005.

[24] F.R. Menter, Two-equation eddy-viscosity turbulence models for engineering applications, AIAA_J. 32 (1994) 1598-605. doi: 10.2514/3.12149.

[25] S. Wang, D.B. Ingham, L. Ma, M. Pourkashanian, Z. Tao, Turbulence modeling of deep dynamic stall at relatively low Reynolds number, J. Fluids Struct. 33 (2012) 191-209. doi: 10.1016/j.jfluidstructs.2012.04.011.

[26] F. Ponta, G. Dutt, An improved vertical-axis water-current turbine incorporating a channelling device, Renewable Energ. 20 (2000) 223-41. doi: 10.1016/S0960-1481(99)00065-8.

[27] M. Shiono, K. Suzuki, S. Kiho, Output characteristics of Darrieus water turbine with helical blades for Tidal Current generations, Proceedings of the 12th International Offshore and Polar Engineering Conference. Kitakyushu, Japan. 26-31 May 2002.

[28] Y. Li, S.M. Calisal, Three-dimensional effects and arm effects on modeling a vertical axis tidal current turbine, Renewable Energ. 35 (2010) 2325-34. doi: 10.1016/j.renene.2010.03.002.

[29] J.H. Strickland, B.T. Webster, T. Nguyen, A vortex model of the Darrieus turbine: an analytical and Experimental Study, J. Fluids Eng. 101 (1979) 500-5. doi: 10.1115/1.3449018. 